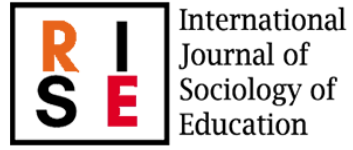

International

Journal of Sociology of Education

\section{Hipatia Press}

www.hipatiapress.com

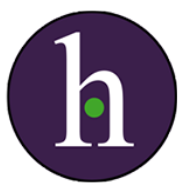

Instructions for authors, subscriptions and further details:

http://rise.hipatiapress.com

\title{
La Educación Secundaria en España en Medio de la Crisis del COVID-19
}

Francisco Javier Aznar Sala ${ }^{1}$

1) Universidad Católica de Valencia, Spain

To cite this article: Aznar Sala, F.J. (2020). La Educación Secundaria en España en Medio de la Crisis del COVID-19, International Journal of Sociology of Education, Special Issue: COVID-19 Crisis and Socioeducative Inequalities and Strategies to Overcome them, 53-78.

http://doi.org/10.17583/rise.2020.5749

To link this article: $h$ ttp://dx.doi.org/10.17583/rise.2020.5749

PLEASE SCROLL DOWN FOR ARTICLE

The terms and conditions of use are related to the Open Journal System and to Creative Commons Attribution License (CC-BY). 


\section{Secondary Education in Spain amid the COVID-19 Crisis}

Francisco Javier Aznar Sala

Universidad Católica de Valencia

(Received: 22 May 2020; Accepted: 7 June 2020; Published: 20 June 2020)

\section{Abstract}

The Spanish educational system has shown that it is not fully prepared for an emergency in the form of online education. In mid-March classes began to be suspended at all educational levels, from infant to university education, with the consequent readjustment of the educational model. From that moment everything has been a challenge and, at the same time, an opportunity to implement educational formats that are possible in the XXI century. Families have had to coordinate with teachers so that the objectives set in the curriculum could be carried out. For schools it has been an unprecedented challenge with the aim that no student is left behind in achieving the proposed objectives. Thus, we present a case study that allows us to understand the organization of a school that can be the object of study for so many other schools that, in the same way, have had to give solutions instantly and provides us with a short-term analysis.

Keywords: pandemic, education, organization, curriculum, objectives 


\section{La Educación Secundaria en España en Medio de la Crisis del COVID-19}

Francisco Javier Aznar Sala

Universidad Católica de Valencia

(Recibido: 22 Mayo 2020; Aceptado:7 Junio 2020; Publicado: 20 Junio 2020)

\section{Resumen}

El sistema educativo español ha demostrado no estar del todo preparado para una emergencia en forma de educación en línea. A mediados de marzo se empezaron a suspender las clases en todos los niveles educativos, desde infantil a la educación universitaria, con el obligado reajuste del modelo educativo. A partir de aquí todo ha supuesto un reto y a la vez una oportunidad de implementar formatos educativos que son posibles en pleno siglo XXI. Las familias se han tenido que coordinar con el profesorado para que los objetivos marcados en el currículo se pudieran desarrollar. Para los centros ha supuesto un desafío sin precedentes con la finalidad primera de que ningún alumno quede atrás en la consecución de los objetivos. Así pues, presentamos un estudio de caso que nos permite comprender la organización de un centro que puede ser objeto de estudio para tantos otros centros que del mismo modo han tenido que dar soluciones al instante y que nos permite un futuro análisis.

Palabras clave: pandemia, educación, organización, currículo, objetivos. 


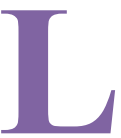

a realidad educativa y social española se ha visto notablemente convulsionada por la pandemia que azota el mundo en forma de coronavirus o Covid-19 y que, en el Sur de Europa, está siendo extremadamente compleja y virulenta. La realidad educativa no ha sido ajena a este problema y, la verdad, nadie estaba realmente preparado para una eventualidad de estas características. Las administraciones públicas han tenido que salir al paso de este escenario sin tener prevista una situación de tal envergadura en un sistema educativo español por modernizar (Gómez, 2009). Desde mediados de marzo la educación presencial se ha visto relegada a un tipo de educación virtual en los hogares, siendo los padres los que han tenido que asumir gran parte de la responsabilidad docente de sus hijos, al menos en lo que respecta a la planificación del trabajo diario enviado por cada profesor y en coordinación con los tutores. Cabe decir que esta modalidad virtual ya venía siendo una posibilidad real que en muchos casos no se implementaba por no dejar de atrás modelos aparentemente más seguros (Gómez \& Cabero, 2004).

Las redes sociales han cobrado un papel fundamental que no ha sido fácil para muchos y hasta que se han ido habilitando distintas formas de trabajar ha tenido que darse un necesario periodo de aprendizaje: "Algunos padres consideran que este modelo ayuda a sus hijos a ser más autónomos e independientes" (Briones, 2003, p.3). Se trata de un formato que ha resultado ser mixto entre la tutorización de los profesores de los distintos centros y el auxilio de los padres. La ayuda familiar resulta de vital importancia para que se garantice el éxito de este sistema, pues es necesario que se revista el hogar de un determinado clima de estudio y de unas determinadas franjas horarias para un trabajo correctamente realizado (Goiria, 2015). La verdad es que no resulta sencillo para muchos, máxime cuando la tensión que se percibe cada día en forma de noticias y de los propios problemas derivados de más de 2 meses de confinamiento y con realidades familiares diversas, agudizan la situación. No obstante, de esta experiencia se pueden extraer algún tipo de resultados positivos que ayuden a corto y medio plazo en el mundo educativo, pues nadie sabe si una situación de estas características se puede volver a repetir en el próximo curso. La educación escolar es capaz de explorar nuevos modelos docentes donde el alumnado cobre mayor protagonismo (Jiménez, 2019a). El presente paradigma educativo ha venido para quedarse y no se trata de una crisis circunstancial. Nuestros alumnos han adquirido en este momento un perfil PLE (Personal Learning Environments) y los PLN (Personal 


\section{Aznar Sala-COVID-19 y Educación Secundaria en España}

Learning Networks), lo que permite un tipo de aprendizaje que facilita a los docentes la educación remota, pues el alumnado tiene acceso a la información según sus propios intereses, objetivos o motivaciones (Rahimi, Van den Berg, y Veen, 2015). La educación no podía quedar al margen de esta realidad que hora se ha potenciado más si cabe. Lo que cabe hacer ahora es adaptar esta realidad o perfil discente hacia una educación de calidad.

Nadie está hablando del fin de la educación presencial, pero sí que estamos en condiciones de señalar que este hecho debe potenciar las herramientas $E$ learning. Las programaciones de los distintos departamentos no incluyen entre sus objetivos todas las posibilidades que brinda este sistema y se hace necesario que se establezcan directrices claras sobre qué hacer en casos como el actual y con qué medios (Ferrer, 2012). Como se indica desde la pedagogía, queda todavía mucho por hacer en línea con modernizar la educación hacia un sistema de gamificación y de interconexión que vaya más allá del aula física (López, 2013). Una planificación de educación permanente en cada una de las asignaturas y en sus programaciones didácticas ayudaría sobremanera a modernizar la educación dentro y fuera del aula. Esta tarea está todavía por hacer y en este momento se constata su urgencia (Morales, Cabero \& Llorente, 2013). Se suceden cambios en el paso de un paradigma formal de educación hacia un modelo Do-It-Yourself (DIY) (Kafai \& Peppler, 2011), donde el aprendizaje traspasa los muros de la escuela y es más eficaz cuando el alumnado consigue desarrollarse en los dos contextos, tanto en el presencial como en el remoto (Hall, 2009). Sin duda, un nuevo reto educativo está servido y nadie podrá abstraerse de tal desafío. En algunos casos ya se han incentivado programas donde se pretende medir el impacto del uso de las nuevas tecnologías y el concepto de red social para incrementar la interacción y el compromiso de los estudiantes (Orts-Escolano et al, 2018). Para ello el profesorado ha de detectar en primer lugar las mejores plataformas e implementarlas cotidianamente en el aula y unidas al desarrollo de cada asignatura. La experiencia piloto se ha puesto en marcha sin esperarlo y es hora de programar desde esta nueva perspectiva. Lo piden los alumnos y, como estamos viendo, lo pide la misma realidad social. Las nuevas tecnologías potencian la experiencia educativa y el proceso de aprendizaje en aquellas asignaturas que han asumido el reto de programar desde esta realidad inesperada. Con todo ello se pretende mejorar la interacción, el compromiso y el proceso de aprendizaje de los alumnos en estas materias. Además, estas experiencias pueden incluirse en las metodologías de b-learning, ya que 
pueden llevarse a cabo en línea como trabajo adicional para los estudiantes. Los resultados de las investigaciones obtenidas son halagüeños y la aplicación de las nuevas tecnologías se aplicará a las técnicas de la programación (OrtsEscolano et al, 2018).

\section{El Caso Educativo Español en Plena Pandemia}

Como sabemos en España la educación está transferida a las Comunidades Autónomas, por lo que las directrices que desde el Ministerio de Educación se han ido trasladando han tenido que ser adaptadas y concretadas por las distintas Consejerías Territoriales (García Garrido, 1993). Es cierto que el Consejo de Universidades (2010) especifica que la formación permanente engloba los tres principales tipos de aprendizajes: el aprendizaje formal, no formal e informal/experiencial. Aún así, en España queda todavía mucho camino por recorrer en el sistema educativo virtual. Autores como Atwell (2007) consideran que el aprendizaje autónomo supone el $80 \%$ del total del conocimiento $\mathrm{y}$, en cambio, son pocos los que se atreven a dar un salto cualitativo que apueste decididamente por este tipo de modelo. Prevalecen los modelos que se consideran más seguros pero que no por ello garantiza mejores resultados pues, la masificación en las aulas unida a la conflictividad, pueden ralentizar un aprendizaje que muchas veces ha de ser atendido por un docente con una excesiva ratio.

Desde el Ministerio de Educación se han ido manteniendo diversas actuaciones en conjunto con los distintos responsables de las autonomías para dar soluciones con la mayor celeridad posible. Para tal fin era imprescindible establecer unos puentes de comunicación lo más agiles posibles con todos los agentes educativos: la administración, los consejeros autonómicos encargados de la educación, centros de primaria y secundaria y el nivel universitario. Pero esta comunicación era prácticamente paralela a la que realizaban [en justa corresponsabilidad] los distintos centros con sus alumnos o con las familias. El reto era de enorme magnitud, dado que no había experiencia sobre ello, pero el objetivo era que nadie quedara atrás. No se podía perder ni un segundo en organización, pues los primeros instantes son decisivos para que los puentes de comunicación queden abiertos y no bloqueados una vez cada alumno estuviera en su casa. A partir de aquí se abriría una tercera vía que ahora desgranaremos y en la que los centros y el profesorado están trabajado con celeridad y a corto plazo, pues urgía dar soluciones y trabajar con un 


\section{Aznar Sala-COVID-19 y Educación Secundaria en España}

alumnado que en pleno siglo XXI es completamente digital (Di Lucca, 2013). El problema es que no se les había encaminado con seriedad por este tipo de educación virtual. La ocasión se presenta siempre como un reto y a la vez como una oportunidad para crecer en las nuevas tecnologías y en nuevos modelos educativos por proyectos que lo docentes han de saber poner en marcha (Jiménez, 2019b).

Aunque de la comparecencia de la ministra del ramo se ha traslado a la opinión pública que se dará un aprobado general para todo el alumnado, los docentes sabemos que esto no es así y que la realidad del aula es mucho más compleja que esto. Realmente no se dijo esto, sino que se dio a entender que los contenidos del tercer trimestre se tenían que relajar y dejar los aspectos más esenciales para darse transversalmente a lo largo del curso 2020-2021 (Farreras, 2020). Es decir, no se trata en este periodo tanto de adelantar contenidos como de afianzar los logros conseguidos en forma de objetivos. Todos los contenidos serán computables y evaluables en miras a lo que cada alumno haya ido realizando a lo largo de los dos primeros trimestres, pero no por ello se pierde la tercera evaluación. Es posible que la mayoría de alumnos promocionen, pero las materias con resultado negativos, se tendrán que superar en el próximo curso. No olvidemos que la educación está influida por los diversos cambios sociales y en sintonía con las nuevas adquisiciones tecnológicas y no es ajena a ella (Resende, 2013). El presente puede suponer en algunos casos un paso hacia adelante más que hacia atrás. Como señalan algunos expertos (Areekul, Ratana-Ubol, \& Kimpee, 2015), este nuevo modelo educativo supondrá un tipo de aprendizaje autónomo que perdure en el tiempo por lo positivo de sus resultados (2015). No podemos perder de vista que nuestros alumnos pertenecen a la generación tecnológica Web 2.0, y tal posibilidad se estaba trabajando a medias en los centros educativos. Aquellos centros que trabajaban desde esta dimensión han visto facilitada su planificación educativa en el confinamiento, el resto han tenido que actualizarse a marchas forzadas, pero en ambos casos este proceso de aprendizaje es ya una realidad imparable.

\section{Metodología}

El proceso metodológico que hemos desarrollado en el presente artículo se ha centrado en diagnosticar cómo se está planificando la educación a distancia ante un reto como el actual. El objetivo principal era comprobar si ningún 
estudiante se quedaba excluido de este modelo a distancia y que todos pueden seguir el proceso educativo a distancia. Para tal fin había que analizar con detalle cómo se ha planificado un centro concreto y cómo se han ido superando situaciones de diversa índole. No olvidemos que cada situación problemática que se genera, en su misma novedad, permite una serie de mejoras que aportan nuevas soluciones (Lewis, 2009). La metodología que hemos utilizado para este trabajo se centra en el Estudio de Caso, pues también estamos inmersos como protagonistas en tal proceso educativo. Al ser un estudio realizado desde el mismo interior del centro educativo se presenta como cualitativa y colaborativa. La mayor fortaleza de esta metodología reside en que nos permite, por medio del registro diario, el seguimiento real de todas las secuencias del alumnado y la coordinación propia que requiere todo un claustro docente con su equipo directivo. Consideramos de este modo conveniente dar la voz a los protagonistas y que se visualice toda la problemática y su posible desarrollo (López, 2016). Las tablas que hemos ido incorporando en el presente estudio son fruto de lo que hemos ido trabajando a lo largo de estos meses desde el centro educativo del cual formamos parte y sobre las que estamos trabajando a diario.

El presente Estudio de Caso se ha desarrollado a lo largo de los meses de marzo, abril y mayo de 2020 dentro del contexto actual de pandemia del COVID-19 que hizo imposible seguir la marcha normal de clases en el centro educativo y tuvo que adaptarse un tipo de educación online. La secuencia de estudio se entregaba de forma digital y se trata de un caso real, aunque el nombre de los informantes permanezca velado por la Ley protección de datos, pero que merece ser estudiado por su particularidad histórica. Todo este proceso de análisis y recopilación de información nos ha permitido hacernos una composición adecuada que ayuda a establecer marcos de trabajo futuros. La reflexión sobre el presente estudio de caso, con datos fehacientes de todo su proceso, permite establecer marcos de mejora de aquellas sinergias que se hayan mostrado como positivas o reforzar otras que sean mejorables. A continuación, se expone una tabla que muestra cuándo y cómo se planificaron las distintas actuaciones: 


\section{Aznar Sala-COVID-19 y Educación Secundaria en España}

Tabla 1

Descripción de los instrumentos de análisis.

\begin{tabular}{ll}
\hline & Relatos de Organización Docente ante el Covid-19 en Secundaria \\
\hline Meses & Descripción \\
Marzo & Reunión de todo el claustro docente ante el inminente cierre de \\
& los centros para establecer pautas Classrroom para educación on- \\
& line. El proyecto Aules de Conselleria todavía no estaba en \\
& marcha y necesitaba de unos 15 días para estar habilitado, por lo \\
& que Classrroom era una solución momentánea. \\
Marzo & Al poseer el centro previamente un dominio en Gmail toda esta \\
& labor de contacto y organización de los alumnos por medio de sus \\
& grupos referenciales resultó cómoda e inmediata. Los alumnos \\
& empezaron desde el primer día a trabajar con sus profesores por \\
& este medio. No se percibió ningún síntoma de descontrol o de \\
& perdida de alumnos. \\
Abril & Se puso en funcionamiento el Plan Mulan desde Conselleria y \\
& todo el profesorado empieza a trabajar desde esta plataforma y a \\
& dar clases virtuales desde entonces. \\
& Se realiza una encuesta a las familias sobre cómo perciben la \\
& marcha del trabajo de los hijos y la relación con el centro. \\
& Todo está en marcha con normalidad virtual y cada alumno puede \\
Mayo & seguir las asignaturas desde casa. Son pocos los alumnos que se \\
& quedan descolgados y las razones técnicas son los menos. En \\
& estos alumnos prevalece más la falta de motivación hacia el \\
trabajo. & Reuniones por Departamentos, por Grupos, Claustros, etc, todo se \\
da según el plan previsto y sin incidencias. Nadie queda atrás y la \\
comunicación interna es perfecta.
\end{tabular}

Los datos que hemos ido incorporando los hemos organizado por fases, tal y como se aconseja (Romero, 2004). El presente método de estudio permite que los distintos datos y fuentes, tanto cualitativas como cuantitativas: 
documentos, registros de archivos, entrevistas directas, observación directa, etc., aporten luz al conjunto del estudio. Los mejores estudios de caso abarcan tanto el lado descriptivo como el explicativo, de modo que ambos se complementan y nos permiten comprender la realidad en su conjunto. Consideramos que la muestra es lo suficientemente representativa dado el conocimiento e interrelación que se tiene con otros centros. Hemos seguido las directrices de investigación y análisis que se recomiendan en el plano metodológico (Martínez-Carazo, 2006), y que se desarrollan en los siguientes puntos:

1. Fase heurística: fase hecha de observación, descripción y reflexión o generalización inductiva.

2. Fase de justificación-confirmación: proceso de comprobación de las hipótesis planteadas con anterioridad.

3. Respecto a su propósito, las investigaciones realizadas a través del método de estudio de caso pueden ser: descriptivas, si lo que se pretende es identificar y describir los distintos factores que ejercen influencia en el fenómeno estudiado; y exploratorias, si a través de las mismas se pretende conseguir un acercamiento entre las teorías inscritas en el marco teórico y la realidad del objeto de estudio.

Los principales supuestos planteados giran en torno a las nuevas posibilidades que se presentan más que a los problemas derivados de la pandemia. Suponemos que de esta realidad pueden surgir soluciones al caso que han venido para quedarse y que eran ya una realidad en potencia, pero no del todo en acto. Así pues, las principales líneas de investigación que nos marcábamos al inicio eran las siguientes:

1. Los centros poseen la capacidad de realizar una enseñanza remota de calidad con sus alumnos, pero esto no se ha puesto todavía en marcha, por lo que requería de una experiencia piloto que ahora se ha dado de forma forzada.

2. Los alumnos son capaces, junto a sus familias, de seguir este modelo educativo y crecer en autonomía y en las diversas competencias educativas. Muchas veces el gran grupo distrae y acomoda la búsqueda de información del alumno. 


\section{Aznar Sala-COVID-19 y Educación Secundaria en España}

3. Los centros son capaces autónoma y organizativamente de realizar proyectos para que ningún alumno quede atrás, pues las posibilidades en forma de herramientas y de recursos humanos son muchos y la profesionalidad de los docentes es elevada.

Se ha efectuado una encuesta que tiene la finalidad de conocer de primera mano la satisfacción de las familias en este periodo de confinamiento en relación al estudio de sus hijos, como aconsejan algunos expertos (Marchesi \& Pérez, 2005). Las encuestas se han realizado desde el centro y hemos tenido acceso a la información hemos ayudado en su confección y distribución. La encuesta se envió por el medio telefónico tokapp al que tienen acceso todas las familias del centro como un medio de comunicación entre el centro y las familias. Se ha enviado a un total de 293 familias y han contestado a la encuesta 265 familias, lo que supone un $90 \%$ del total. El total de los padres tiene a sus hijos escolarizados en Secundaria, Bachillerato y Formación Profesional. El 50\% están en educación secundaria, el 27\% cursan 1 y 2 de bachillerato y un $23 \%$ está realizando formación profesional. Todos ellos pertenecen a un centro público que abarca tres localidades que suman unos 6000 habitantes.

Se trata de un medio rural formado por familias trabajadoras de clase media. El instrumento de la encuesta que se ha utilizado se ha basado en un cuestionario anónimo e individual que ha llegado a cada familia a través del programa tokapp school entre los días laborables del 20 al 24 de abril de 2020 y desde este programa se ha realizado la recogida de datos de la encuesta que hemos analizado y referenciado desde el programa Excel. Los 4 ítems que se las han preguntado giraban en torno a la satisfacción de la conexión de sus hijos con los profesores y el grado de implicación de sus hijos con los trabajos que se les pedían. Las variables giraban en torno a las siguientes preguntas y las posibles respuestas se tenían que elegir entre tres posibles variables según el grado de conformidad: Bueno, Adecuado, Insuficiente:

1. Conexión a la red virtual.

2. Valoración del trabajo de los profesores y seguimiento del alumnado.

3. Organización del centro en comunicación con las familias.

4. Opinión sobre el seguimiento de sus hijos y trabajo en casa. 


\section{Análisis de Caso del Trabajo Realizado en un Centro de Secundaria}

Para que se pueda realizar un tipo de estudio donde los alumnos no se queden atrás, especialmente en momentos como el actual, es imprescindible una buena organización en red y un buen trabajo de planificación por parte de todo el claustro docente conjuntamente con su equipo directivo. La experiencia está demostrando que trabajar en línea posee una serie de ventajas que no éramos capaces de visualizar en toda su amplitud. Las posibles deficiencias se han tenido que ir solucionado a medida que se producían y ello ha permitido un perfeccionamiento progresivo de las actividades. En un mundo tan interconectado como el nuestro poner en marcha la competencia de "aprender a aprender" resulta una tarea imprescindible para que el alumno crezca en autonomía y creatividad. El objetivo del centro desde el inicio era claro: "Que cap alumne quede enrere" (Que ningún alumno se quede atrás). El programa escogido por la administración valenciana para que los docentes pudieran trabajar con sus alumnos ha sido el Pla MULAN que incluía la plataforma Moodle de Aules, para el teletrabajo con archivos y corrección, y la Webex meetings con posibles conferencias con los distintos grupos. El Pla MULAN es un método unificado de docencia digital diseñado para impartir, de manera no presencial, las asignaturas y materiales correspondientes a las diversas etapas escolares de la ESO y Bachillerato. Un plan diversificado de las nuevas tecnologías que lo constituyen y con un objetivo único: garantizar la docencia en línea al máximo alumnado posible y ayudar al profesorado en el acceso a estas herramientas.

La nueva metodología tardó un par de semanas en estar al alcance de todos y era necesario que se implementara lo antes posible para poder trabajar con el alumnado. Con el fin de subsanar posibles desigualdades entre los alumnos de Secundaria, el día 1 de abril Conselleria d'Educació $i$ Ciència de la Comunitat Valenciana, notificó que se iba a llevar a cabo una actuación para mejorar la formación a distancia de los alumnos y de las familias con menos recursos o de aquellos que vivían en zonas con peor conexión a internet. Con esta finalidad se repartieron una serie de tabletas digitales que llevaban incorporadas las correspondientes tarjetas SIM de línea con datos de alta capacidad (40 GB mensuales). Esta medida procuraba que nadie se quedara excluido. Para determinar el número de las tabletas digitales por centro se baremó el total del alumnado y el índice socioeconómico y cultural (ISEC) de cada zona o pueblo. Así lo aconsejan los distintos informes $P i s a$ en relación a 


\section{Aznar Sala-COVID-19 y Educación Secundaria en España}

una serie de criterios que están publicados a todos los efectos y desde los que se han realizado algunos estudios (Saz, 2006). En el caso concreto que estamos estudiando se asignaron un total de 14 dispositivos para que se trasladaran a los alumnos más necesitados. Para tal fin las familias tenían que rellenar una solicitud que se abría en la plataforma digital el día 2 de abril a las 9:00 horas y se cerraba el día 3 de abril a las 23:00. Era imprescindible averiguar con celeridad quiénes eran los posibles beneficiarios de estas herramientas digitales y alguna más que había puesto el centro para ampliar la cobertura. La misma jefa de estudios realizó una petición formal el lunes 30 de marzo a todas las familias con la inestimable cooperación de todos los tutores, el claustro de profesores, el departamento de orientación y los servicios sociales de la localidad que ayudaron a establecer una serie de criterios lo más objetivos posibles.

De esta forma se localizaron con prontitud los alumnos idóneos para recibir esta imprescindible herramienta con la finalidad trabajar desde casa y no quedar excluidos en un modelo que procura ser lo más inclusivo posible desde hace tiempo (Escudero \& Martínez, 2011). Como señala Casanova, el modelo educativo del futuro será inclusivo o fracasará (2011). De este modo, el número de alumnos que pueden seguir el estudio dirigido desde casa se ha universalizado y pocos quedan excluidos de la conexión a internet y del correspondiente feedbak con sus profesores (en el programa destinado a tal fin de Aules y Webex). El material queda asignado en calidad de préstamo a los alumnos hasta que pase esta contingencia y se devuelvan al centro una vez se reprendan las clases de forma normalizada. Lo más importante es que prevalezca la inclusión ante cualquier contingencia que pueda darse en el modelo educativo (Fernández \& Malvar, 2019). Se ha realizado un trabajo todavía más somero de estudio para ampliar la cobertura y se han podido repartir incluso algunos portátiles más que el centro tenía en propiedad para el trabajo cotidiano. Como señalan los pedagogos (Ponerulappan y Thilagavathy, 2014), nuestra sociedad posee el poder innato de adaptarse y resolver una serie de problemas de los que no éramos plenamente consciente antes de afrontarlos. Da la sensación de que "la escuela tradicional queda atrasada con respecto a los adelantos de la psicología y de la ciencia [...] y está al margen de las profundas transformaciones que se producen" (Martorell, 1965, p.10). La educación remota es una posibilidad importante: "ya no existe un tiempo y lugar determinado para aprender, por lo que cada vez es más urgente una nueva cultura de aprendizaje que rompa con los 
métodos tradicionales que no están dando la respuesta adecuada a la actual realidad educativa" (Valdés \& Gutiérrez, 2018, p.6). Para que esto pueda darse es necesario realizar una adecuada planificación en los centros escolares.

Tradicionalmente los métodos educativos en los distintos centros de educación de secundaria están ligados a la lección magistral y con pocas conexiones digitales o de E-learning que, paradójicamente, son plenamente posibles y muchos las utilizan. Una de las situaciones más problemáticas desde el inicio ha sido la necesidad de tener a todo el conjunto de alumnos habilitados en red. Esta era la tarea principal y más ardua, pues se han ido dando algunos casos donde el alumno no respondía a las primeras actividades diseñadas por los distintos departamentos. Una vez localizado, mediante un mail circular a todo el profesorado por parte de cada tutor de curso, se han realizado unos informes para que desde jefatura de estudios se subsanara cuanto antes esta dificultad -en contacto con las familias respectivas-. En algunos casos se dan circunstancias personales, en otros algo de desinterés por parte del alumnado o las familias y en otros una posible brecha digital que es necesario subsanar de inmediato por parte del centro educativo. Como señalan algunos expertos, el estatus socioeconómico familiar, medido por la cultura y el nivel económico de los padres del alumno, pude influir influye en el acceso de la Web 2.0 (Márquez y Rincón, 2013). En la tabla que sigue a continuación podemos visualizar un ejemplo de cómo se contactó con las familias y el medio, para que sus hijos, como el resto de sus compañeros, se conectaran cuanto antes a la red y poder ayudarles en las posibles dificultades de conexión, falta de material digital o de motivación. 


\section{Aznar Sala-COVID-19 y Educación Secundaria en España}

Tabla 2

Selección de tabla que permite visualizar con qué medios se ha contado para contactar con las familias inicialmente

\begin{tabular}{lccc}
\hline \multicolumn{1}{c}{ Seguimiento asignatura } & Fecha & Vía & Pariente \\
\hline Valenciano & $23 / 03 / 20$ & Tokapp & \\
Valenciano & $23 / 03 / 20$ & Teléfono & Abuela \\
Física y Química & $23 / 03 / 20$ & Tokapp & \\
Inglés & $23 / 03 / 20$ & Tokapp & \\
Economía & $23 / 03 / 20$ & Teléfono & Madre \\
Matemáticas & $23 / 03 / 20$ & Tokapp & \\
Geografía e Historia & $23 / 03 / 20$ & Tokapp & \\
Tecnología & $23 / 03 / 20$ & Teléfono & Padre \\
Física y Química & $24 / 03 / 20$ & Teléfono & Madre \\
Matemáticas & $24 / 03 / 20$ & Tokapp & \\
\hline
\end{tabular}

La metodología del trabajo diario enviado por cada profesor a sus alumnos ha resultado ser uno de los principales problemas que han salido a la luz en esta nueva coyuntura. Los padres -en general- han manifestado su malestar a los tutores por la excesiva carga de trabajo que cada día se les enviaba a sus hijos y que en medio de una situación compleja les tenía pegados al ordenador o al escritorio todo el día y pendientes de sus hijos. La falta de planificación de los deberes por parte del profesorado ha sido uno de los principales escollos que se han tenido que subsanar en este confinamiento. El problema estaba latente, pero en esta coyuntura se ha hecho patente, por lo que falta una verdadera planificación del trabajo semanal en el conjunto del profesorado antes, durante y después de este momento. Cabe decir que la cuestión de los deberes y trabajos que se mandan a los alumnos es una de las cuestiones más discutidas en el actual panorama educativo y que ahora está sobre la mesa. Hay indicios de que muchos estudiantes hacen los deberes escolares no por el interés o entusiasmo que les producen, sino más bien por un sentido del deber o del deseo de agradar o la evitación del castigo (Walker, Hoover-Dempsey, Whetselm y Green, 2004).

Conociendo la realidad de los alumnos en un momento de estrés y de incertidumbre, no tenía ningún sentido establecer e incrementar pautas de trabajo como si el alumno estuviera en el aula en un periodo normal. El trabajo en casa pedía ser racionalizado para que quedara espacio para la actividad física y un estilo de trabajo lo más atractivo posible. El estudio continuado en casa pide ser formativo, pedagógico y que incluya posibles variables (Cabrera 
\& Fariñas, 2019). Con esta finalidad se diseñó desde el centro una tabla semanal que mostrara holísticamente todo el trabajo planteado para los alumnos de cada grupo. El trabajo no podía superar las 12 o 14 horas en la ESO y las 17 o 18 en Bachiller. Hay un máximo de trabajo semanal estipulado en horas y por asignatura. Esta organización ha venido como efecto de la pandemia, pero ha venido para quedarse, pues antes cada profesor ponía sus tareas con independencia del resto de compañeros y ahora se ha visto la necesidad de hacerlo conjuntamente. Se puede hacer mediante el programa que ofrece Drive y se rellena al final de cada semana para que los lunes esté todo organizado.

Tabla 3

Selección de tabla que permite la organización semanal del trabajo del alumno en cómputo global

\begin{tabular}{ccccccc}
\hline 4t ESO & Lunes & Martes & Miércoles & Jueves & Viernes & \\
\hline MATERIA & $20 / 04$ & $21 / 04$ & $22 / 04 / 20$ & $23 / 04$ & $24 / 04$ & PA \\
EF & 0,25 & $0{ }^{\prime} 25$ & $0{ }^{\prime} 25$ & & 0,25 & $45^{\prime}$ \\
& $\left(15^{\prime}\right)$ & & & & & \\
CLL & $0,5\left(30^{\prime}\right)$ & $1\left(60^{\prime}\right)$ & $0{ }^{\prime} 5$ & 1 & 0,5 & 2,5 \\
VAL & 1 & 1 & & & 1 & 3 \\
GH & $0{ }^{\prime} 5$ & & 0,5 & & 0,5 & 1,5 \\
ING & & $0{ }^{\prime} 5$ & & 0,5 & & $1 \mathrm{~h}$ \\
FL & $0{ }^{\prime} 5$ & & 0,5 & & 0,5 & 1,5 \\
MAT & 1 & 1 & 1 & 1 & & 3 \\
FQ & & & & 1 & 1 & 2 \\
Total diario & 2,75 & 3,75 & 2,75 & 3,5 & 3,25 & 14,5 \\
\hline
\end{tabular}

La organización en una situación como la que estamos viviendo es fundamental. En la planificación del trabajo semanal y el seguimiento de profesores y tutores de cada alumno se juega el que nadie quede atrás en la formación. Con este propósito se confeccionan todas las tablas y se realizan asiduas reuniones virtuales por Webex. La finalidad es analizar cómo va cada alumno y si es necesario tomar algún tipo de medida en aquellos que se retrasan. La realidad es que el trabajo está siendo muy efectivo y la coordinación constante de todo el claustro permite que casi el $85 \%$ de los alumnos estén conectados. La tabla que sigue se rellena al final de cada semana; es decir, los viernes, para que cada alumno y profesor puedan 


\section{Aznar Sala-COVID-19 y Educación Secundaria en España}

planificar también las sesiones síncronas en que se conectaran con el profesor para dar la calase y resolver dudas por la cámara:

Tabla 4

Selección de tabla que permite visualizar y planificar las clases síncronas

\begin{tabular}{|c|c|c|c|c|c|}
\hline & Lunes & Martes & Miércoles & Jueves & Viernes \\
\hline 1 & $\begin{array}{c}\text { Geografía e } \\
\text { Historia }\end{array}$ & $\begin{array}{c}\text { Geografía e } \\
\text { Historia }\end{array}$ & $\begin{array}{l}\text { Educación } \\
\text { Física }\end{array}$ & Valenciano & $\begin{array}{l}\text { Física y } \\
\text { Ouímica }\end{array}$ \\
\hline 2 & Filosofía & Valenciano & $\begin{array}{l}\text { Física y } \\
\text { Química }\end{array}$ & Economía & Latín \\
\hline & Descanso & & & & \\
\hline 3 & & & Valenciano & $\begin{array}{c}\text { Geografía e } \\
\text { Historia }\end{array}$ & Matemáticas \\
\hline 4 & $\begin{array}{l}\text { Matemáticas } \\
\text { Descanso }\end{array}$ & Inglés & Inglés & Inglés & \\
\hline 5 & $\begin{array}{c}\text { Religión / } \\
\text { Valores }\end{array}$ & Tecnología & Filosofía & Tecnología & Filosofía \\
\hline 6 & & Música & & & Música \\
\hline
\end{tabular}

Además, ha sido necesario establecer una pauta de reuniones semanales que permitan al profesorado situarse ante la realidad académica de cada uno de sus alumnos. Algunos de ellos llevan asignaturas pendientes del curso pasado y es necesario adaptar el trabajo para que se pueda superar dicha asignatura. Para ello se ha numerado con una serie de ítems y colores el seguimiento por cursos y por asignaturas. La finalidad es que cada profesor posea una visión holística de la realidad de cada alumno y el tutor pueda mediar de inmediato en aquellos casos que lo requieran. La tabla ha sido de enorme ayuda al permitir una actuación rápida según la información que volcaba cada profesor y alumno por alumno. Este tipo de actuaciones están permitiendo que la labor educativa no se resienta y que la falta de encuentro personal no redunde en una especie de distanciamiento. 
Tabla 5

Selección que permite visualizar la realidad académica de cada alumno

\begin{tabular}{|c|c|c|c|c|c|c|c|c|c|c|c|c|}
\hline PN & $\mathrm{GH}$ & $\mathrm{CL}$ & IN & MA & $\mathrm{EF}$ & VA & EC & LA & FQ & BG & TE & FI \\
\hline \multirow{5}{*}{ FQ } & 1 & 1 & 1 & 1 & $\mathrm{CO}$ & 1 & 2 & 1 & 1 & 1 & 1 & 2 \\
\hline & 2 & 2 & 2 & 2 & 2 & 2 & 1 & 2 & 1 & 2 & 1 & 1 \\
\hline & 2 & & 2 & 2 & 3 & 2 & 2 & 3 & 3 & 2 & 3 & 3 \\
\hline & 1 & 1 & 1 & 2 & 1 & 2 & 2 & 2 & 2 & 1 & 2 & 2 \\
\hline & 1 & 1 & 1 & 1 & 1 & 1 & 1 & 1 & 1 & 1 & 1 & 1 \\
\hline \multirow[t]{2}{*}{ M } & 2 & 2 & 2 & & $\mathrm{CO}$ & 2 & 2 & 2 & 1 & 2 & 2 & 2 \\
\hline & 1 & 1 & 1 & 1 & 1 & 1 & 1 & 1 & 1 & 1 & 1 & 1 \\
\hline \multirow[t]{2}{*}{$\mathrm{GH}$} & & 2 & 2 & 1 & $\mathrm{CO}$ & 2 & 2 & 3 & 2 & 2 & 2 & 3 \\
\hline & 2 & 1 & & 1 & 1 & 2 & 1 & 1 & 2 & 2 & 2 & 2 \\
\hline M & 2 & 2 & & 3 & 2 & 2 & 3 & 3 & 3 & 2 & 3 & 2 \\
\hline \multicolumn{5}{|c|}{ No la cursa } & $\mathrm{CO}$ & \multicolumn{3}{|c|}{ Convalida } & 2 & \multicolumn{3}{|c|}{ En ocasiones } \\
\hline \multicolumn{5}{|c|}{2 evaluación suspensa } & $\mathrm{PC}$ & \multicolumn{3}{|c|}{$\begin{array}{l}\text { Pendiente de } \\
\text { convalidar }\end{array}$} & 3 & \multicolumn{3}{|c|}{$\begin{array}{l}\text { No se conecta } \\
\text { nunca }\end{array}$} \\
\hline \multicolumn{5}{|c|}{1 evaluación suspensa } & 1 & \multicolumn{3}{|c|}{$\begin{array}{l}\text { Sigue bien los } \\
\text { temas }\end{array}$} & 4 & \multicolumn{3}{|c|}{$\begin{array}{l}\text { Problemas en la } \\
\text { red }\end{array}$} \\
\hline
\end{tabular}

En el apartado de metodología hemos explicado cómo se realizó una encuesta por parte del centro para conocer el grado de satisfacción de las familias en relación al estudio asistido de sus hijos y la implicación del profesorado. Se les preguntó en los niveles de ESO, Bachiller y Formación Profesional, el grado de satisfacción en diversos ámbitos para paliar posibles dificultades y con la finalidad de dar la voz a los padres para conocer su opinión y mejorar deficiencias. El primer ítem versaba sobre cuál era la realidad de las familias en relación a las herramientas necesarias para que sus hijos puedan seguir el curso y no quedaran aislados. Los ítems poseen tres niveles de grado de satisfacción en cada uno de los niveles académicos: Bueno, Regular, Malo. Podemos comprobar que el grado de conexión de los alumnos, después del esfuerzo inicial realizado por el centro, es altamente valorado por las familias, pues prácticamente nadie se ha quedado atrás en dispositivos para poder seguir las clases con normalidad. Destacan positivamente los niveles de la ESO y Bachillerato con altos índices de conexión a las clases. En cambio, se dan niveles más bajos en Formación Profesional, pero es necesario advertir que muchos de los chicos y chicas que 


\section{Aznar Sala - COVID-19 y Educación Secundaria en España}

cursan estos nieles viven en otras poblaciones, lo que ha dificultado el acceso a estas familias:

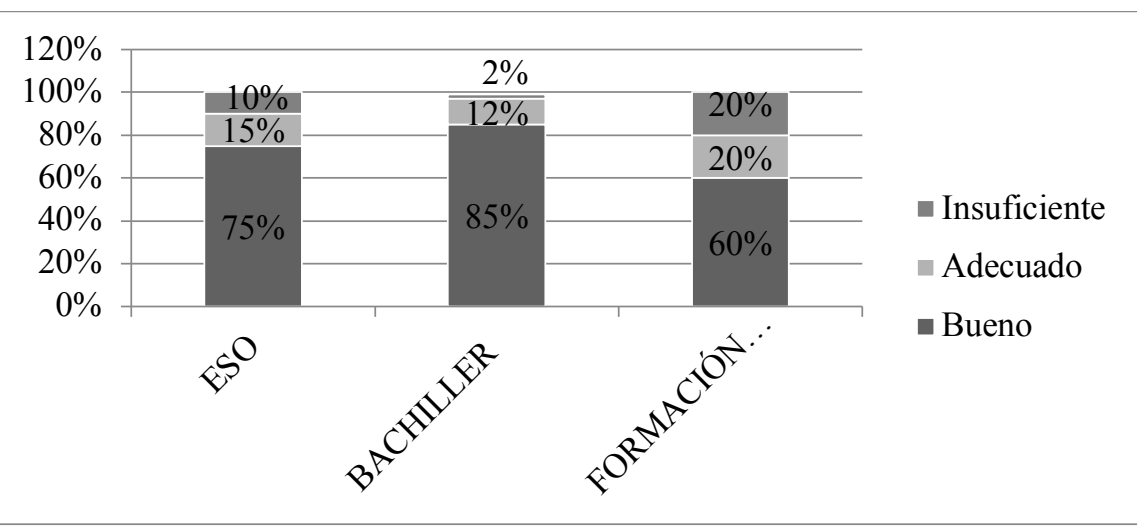

Figura 1. Posibilidad de los alumnos de conectarse a internet desde sus casas. Pregunta 1. ¿Sus hijos pueden seguir las clases y el trabajo desde casa de forma habitual y desde la conexión a internet?

El segundo ítem que se ha valorado por parte de las familias gira en torno al trabajo y seguimiento del profesorado del trabajo de sus hijos. Además de la planificación del trabajo semanal y la carga horaria que siguen sus hijos en cada una de las asignaturas. Es necesario explicar que esta encuesta se realizó después de unos primeros compases de incertidumbre por parte de todos y de la posterior adecuación del trabajo semanal como se puede comprobar en las Tablas 2 y 3 . Es reseñable el hecho de que prácticamente todos los padres consideran positiva la implicación de los profesores. De este modo los porcentajes de valoración del trabajo docente son positivos, teniendo en cuenta que independientemente de la adquisición o no de ordenadores o tabletas que peritan el trabajo del $100 \%$ de los alumnos, se han buscado alternativas para hacerles llegar el trabajo del modo que fuera. En este sentido, el equipo directivo y los distintos tutores han realizado un trabajo encomiable. Hay que considerar que los padres pueden comprobar in situ cómo se conectan cada día los profesores de las distintas asignaturas con sus hijos, de ahí que el grado de compromiso con el alumnado se valora: 


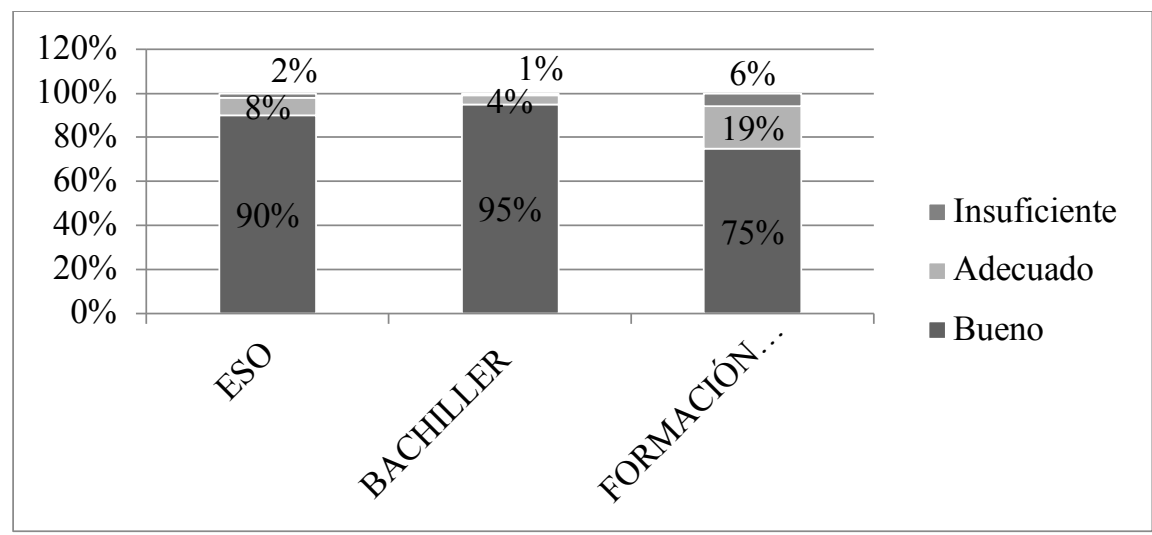

Figura 2. Valoración del seguimiento del profesorado a los alumnos desde la óptica de las familias: Pregunta 2. ¿Considera que los profesores han estado atentos a las necesidades educativas de sus hijos?

Respecto al tercer ítem que se preguntó a las familias sobre la organización del centro en comunicación con las familias los resultados han sido también clarificadores y han arrojado mucha luz sobre cómo han percibido las familias la cercanía del centro en estos momentos de incertidumbre. Las familias son plenamente conscientes del esfuerzo que se ha realizado para que ninguno de sus hijos quede incomunicado en su casa en este periodo de confinamiento que busca proteger la salud de todos ante una emergencia inesperada. La satisfacción es bastante generalizada, aunque algunos padres consideran que era mejorable o incluso escasa o fuera de tiempo, pero son los menos. La valoración acerca de la comunicación ha sido también positiva: 


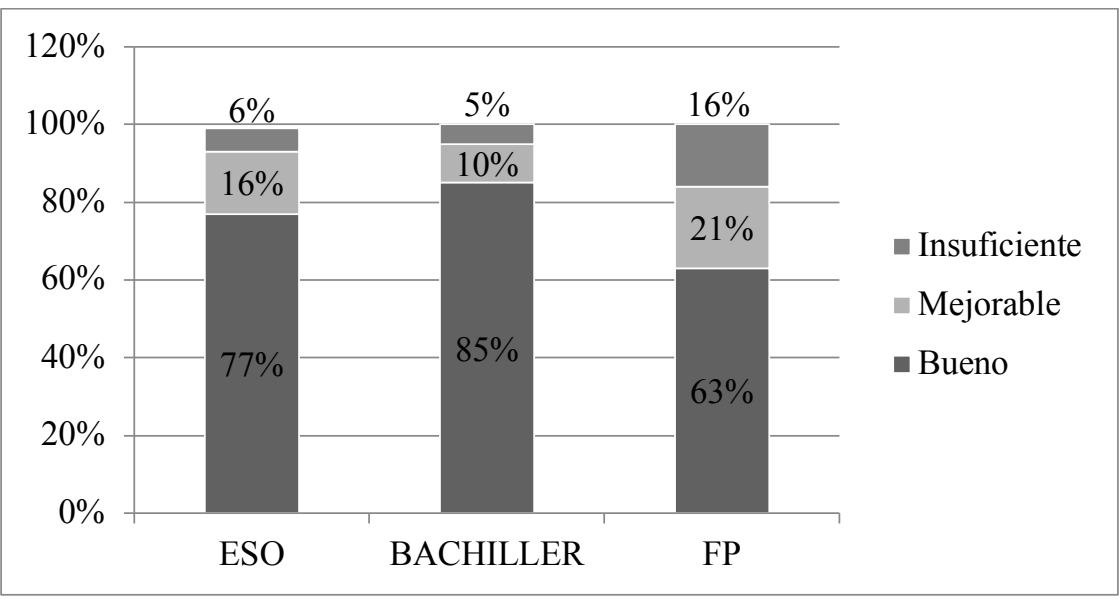

Figura 3. Opinión de la información que ha facilitado encentro a las familias en este periodo de confinamiento. Pregunta 3. ¿Considera que la información que les ha facilitado en centro ha sido la adecuada y justa en los tiempos?

Respecto al aprovechamiento del trabajo en casa, las opiniones son más dispares en una tipología de alumnos que no están del todo entrenados para el trabajo autónomo. El grado de satisfacción es inferior al resto de ítems, pues los padres observan en porcentajes significativos que sus hijos no están aprovechando del todo bien el tiempo de estudios. Naturalmente es más elevada esta sensación en los ciclos de formación profesional $(26 \%)$ y en la ESO $(21 \%)$, debido al tipo de metodología más práctica que se ha seguido durante el curso y que ahora no ha sido fácil de adaptar a esta nueva coyuntura en FP y por falta de madurez y autonomía en los primeros cursos de la ESO. 


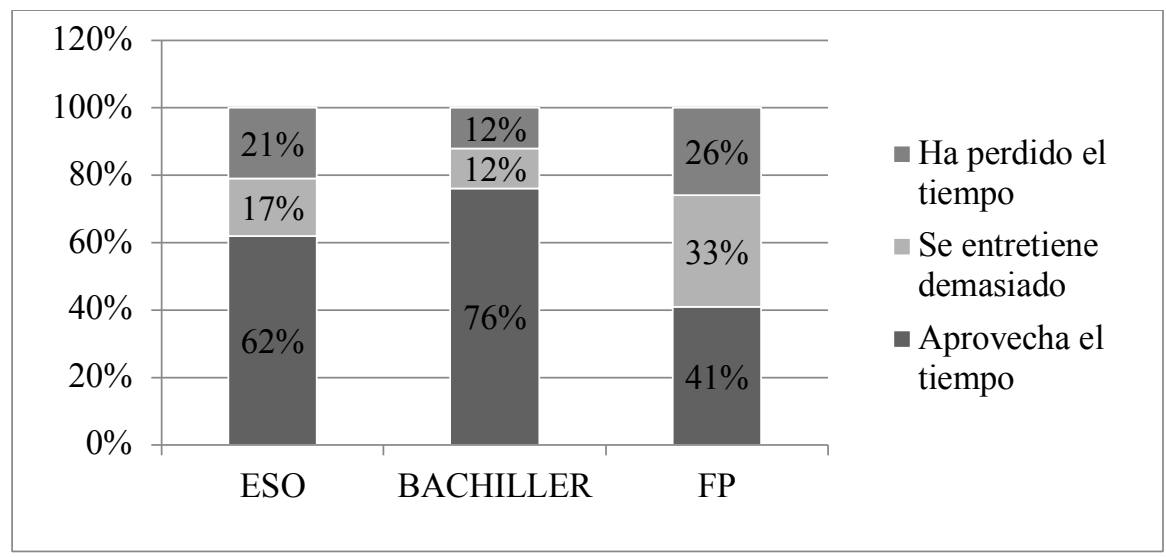

Figura 4. Opinión sobre el seguimiento del aprovechamiento del estudio de sus hijos durante este periodo excepcional. Pregunta 4. ¿Considera que su hijo/a ha sacado provecho diario del trabajo que tenía que realizar en casa o cree que se ha perdido mucho tiempo?

\section{Conclusiones}

La actual pandemia en forma de coronavirus que está azotando el mundo está siendo especialmente virulenta en el Estado Español. Toda esta realidad ha cambiado brusca e inesperadamente el desarrollo natural de todas las actividades que se realizaban en nuestro país. El ámbito educativo se ha visto especialmente afectado por esta crisis, al tiempo que con claridad se ha visibilizado que no había ningún plan alternativo a la educación presencial. Algunos autores ya venían advirtiendo de la necesidad de desarrollar planes de educación a distancia merced a las nuevas posibilidades que ofrecen las nuevas tecnologías. Es más, esta metodología a distancia posee una serie de cualidades que era bueno poner en marcha, como ya ha sido reflejado en el marco teórico. El hecho mantener a los alumnos en sus casas y tener que cerrar los centros desde marzo, ha supuesto un notable desafío a un modelo educativo centrado en las clases presenciales y la falta de potenciación de las nuevas tecnologías aplicadas a la enseñanza. Los distintos centros y claustros de profesores han tenido que salir al paso incluso antes de que la Administración les ofreciera las soluciones adecuadas. Como dato positivo es 


\section{Aznar Sala-COVID-19 y Educación Secundaria en España}

justo decir que ha quedado de relieve el alto grado de profesionalidad docente y de entrega a su trabajo, vivido este como vocación y servicio social. Además, los centros han tenido que organizarse de forma que ningún alumno quedara excluido y pudiera seguir el método de clases síncronas (clases online) o trabajo asíncrono (deberes y trabajos) desde casa. Los esfuerzos se han centrado en habilitar dispositivos para aquellas familias que no disponían de ellos y en coordinación con la Administración, para que la brecha digital no ahondara más en las diferencias entre las familias.

Por el estudio realizado podemos concluir que se han abierto nuevas sendas y se han potenciado modelos que era necesario implementar y que en muchos casos estaban dormidos (como el teletrabajo). Además, la educación por plataformas y por medio de la formación continua en red ha experimentado un notable auge. No es posible que en pleno siglo XXI el modelo educativo siga pivotando en un porcentaje tan alto sobre la educación presencial y la falta de autonomía y motivación del alumnado. Hemos realizado un estudio de caso en un centro de la comarca de la Ribera Alta en la provincia de Valencia, que nos ha permitido comprender desde un estudio directo cómo se han ido solucionando los problemas a medida que se iban suscitando en miras a la inclusión de todo alumno. Sorprendentemente este modelo de enseñanza remota ha tenido números de seguimiento muy positivos, como se describe en las tablas: alrededor del $85 \%$ de los alumnos han seguido el trabajo propuesto por los profesores y las clases síncronas por medio de Webex. Es más, el grado de satisfacción de las familias es elevado y se ha puesto de relieve el buen nivel de profesionalidad docente de la Educación Pública. El seguimiento de cada uno de alumnos y sus posibles problemas, la dotación de materiales para que ningún alumno quede atrás, la planificación semanal de cada asignatura en una visión de conjunto, las constantes reuniones entre el equipo docente con la dirección del centro, han sido algunos de los aspectos que han permitido la inclusión de todos y una coordinación efectiva. Las programaciones de aula no estaban preparadas para un plan alternativo como es la educación a distancia; sin embargo, al implementar de forma real las nuevas tecnologías, ha surgido la necesidad de un tipo de programación que contemple dos alternativas posibles: educación presencial y remota. De hecho, a partir de septiembre se ha de programar desde esta dimensión y teniendo en cuenta que los contenidos que no se hayan podido completar en este curso se darán en el mes de septiembre. 


\section{Referencias}

Areekul, C., Ratana-Ubol, A., \& Kimpee, P. (2015). Model development for strengthening social capital for being a sustainable lifelong learning society. Procedia-Social and Behavioral Sciences, 191, 1613-1617. http://doi.og/10.1016/j.sbspro.2015.04.508

Attwell, G. (2007). Personal Learning Environments-the future of eLearning? Elearning papers, 2(1), 1-8.

https://www.researchgate.net/publication/228350341

Briones, I. M. (2003). ¿La escuela en casa o la formación de la conciencia en casa? Revista General de Derecho Canónico y Derecho Eclesiástico del Estado, 3, 3.

Cabrera Albert, J. S., \& Fariñas León, G. (2019). El estudio de los estilos de aprendizaje desde una perspectiva vigostkiana: una aproximación conceptual.

Casanova, M. A. (2011). La educación inclusiva: un modelo de futuro. Wolters Kluwer.

Di Lucca, S. (2013). El comportamiento actual de la Generación Z en tanto futura generación que ingresará al mundo académico. Universidad de Palermo, Italia.

Escudero, J. M., \& Martínez, B. (2011). Educación inclusiva y cambio escolar. Revista iberoamericana de educación, 55(1), 85-105.

Farreras, C. (2020). Celaá: "No corresponde al Ministerio fijar un número de suspensos para repetir”. La Vanguardia.

https://www.lavanguardia.com/vida/20200417/48574247318/celaacurso-escolar-repeticiones-suspensos.html

Fernández Tilve, M. D., \& Malvar Méndez, M. L. (2019). Las competencias emocionales de los orientadores escolares desde el paradigma de la educación inclusiva. Revista De Investigación Educativa, 38(1), 239257. https://doi.org/10.6018/rie.369281

Ferrer, E. M. (2012). Gamificación y e-Learning: un ejemplo con el juego del pasapalabra. In EFQUEL Innovation Forum (pp. 137-144).

García Garrido, J. L. (1993). Sistemas educativos de hoy. Dykinson.

Goiria, M. (2015). 10 tópicos sobre la Homeschool. CreateSpace Independent Publishing Platform.

Gómez, A. M. (2009). Enseñanza por Competencias. La orientación actual 
76 Aznar Sala-COVID-19 y Educación Secundaria en España

del sistema educativo español. Revista de Educação, 1(1), 7-22. Gómez, J. I. A., \& Cabero, J. (2004). Educar en red. Internet como recurso para la educación. Pixel-Bit. Revista de Medios y Educación, (22), 115.

Hall, R. (2009). Towards a Fusion of Formal and Informal Learning Environments: the Impact of the Read/Write Web. Electronic Journal of e-Learning, 7(1), 29-40. http://www.ejel.org

Jiménez Rodríguez, M.A. (2019a). El diseño de unidades didácticas hoy.

Tirant Humanidades.

Jiménez Rodríguez, M.A. (2019b). Programar al revés. Narcea.

Kafai, Y. B., \& Peppler, K. A. (2011). Youth, technology, and DIY:

Developing participatory competencies in creative media production.

Review of research in education, 35(1), 89-119.

http://doi.og/10.3102/0091732X10383211

Lewis, C. (2009). What is the nature of knowledge development in lesson study? Educational Action Research, 17(1), 95-110.

http://doi.og/10.1080/09650790802667477

López, I. (2016). “Resignificant” la formació inicial de mestres:

l'aprenentatge com a projecte vital (tesis doctoral, Universidad de

Lleida, España). http://www.tdx.cat/handle/10803/401718

López, M. (2013). Los MOOC/COMA: un nuevo reto educativo para el siglo XXI. Una metodología didáctica para el aprendizaje en línea. Virtualis, 4 (8), 85-103.

Marchesi, A., Pérez, E. M., \& Educativo, A. (2005). Opinión de las familias sobre la calidad de la educación. Centro de Innovación Educativa CIE-FUHEM. http://www.fuhem.

es/media/educacion/File/encuestas/Opinion_de_las_familias_sobre_la calidad_de_la_educacion_Encuesta_completa.pdf

Márquez, L., \& Rincón, D. (2013). Brecha digital: uso de internet entre docentes y alumnos universitarios. Revista Negotium, (24), 86-108.

Martínez Carazo, P. C. (2011). El método de estudio de caso Estrategia metodológica de la investigación científica. Revista científica Pensamiento y Gestión, (20).

Martorell, A. (1965) Cómo realizar prácticamente una Escuela Nueva. Tiempo de hacer.

Morales Lozano, J. A., Cabero Almenara, J., \& Llorente Cejudo, M. D. C. 
(2013). Contributions to e-Learning from a Best Practices Study at Andalusian Universities. RUSC: Universities and Knowledge Society Journal, 10(1), 226-239.

Orts-Escolano, S., Cazorla, M., Gómez-Donoso, F., Navarrete Sánchez, J., \& Viejo Hernando, D. (2018). Análisis y evaluación de plataformas online basadas en la gamificación y redes sociales para el desarrollo de habilidades como programador.

Rahimi, E., Van den Berg, J., \& Veen, W. (2015). Facilitating student-driven constructing of learning environments using Web 2.0 personal learning environments. Computers \& Education, 81, 235-246. http://doir.org/10.1016/j.compedu.2014.10.012

Resende, T. F. (2013). “Aprender a conhecer” na sociedade da informação: o papel específico da escola e os seus desafíos. RASE: Revista de la Asociación de Sociología de la Educación, 6(2), 289-301. https://dialnet.unirioja.es/servlet/articulo? codigo $=5144565$

Romero, S. (2004). Aprender a construir proyectos profesionales y vitales. Revista Española de Orientación y Psicopedagogía, 15(2), 337-354. http://doir.org/10.5944/reop.vol.15.num.2.2004.11637

Sarabia Sánchez, F. J. (1999). Metodología para la investigación en marketing y dirección de empresas, Madrid, Pirámide.

Saz, M. (2006). Influencia del nivel socioeconómico y cultural en el rendimiento de los estudiantes de tercero básico y graduandos del año 2006. Dirección General de Evaluación e Investigación Educativa.

Valdés, V. \& Gutiérrez, P. (2018). Las Urgencias Pedagógicas en la sociedad del aprendizaje y el conocimiento. Un estudio para la reflexión sobre la calidad en el nuevo modelo educativo. Multidisciplinary Journal of Educational Research, (8), 1-28. http://doir.org/10.17583/remie.2018.3199

Walker, J.M.T., Hoover-Dempsey, K.V., Whetselm, D.R., y Green, C.L. (2004). Parental involvement in homework: A review of current research and its implications for teacher, afterschool program staff, and parent leaders. Cambridge: Harvard Family Research Project. 
78 Aznar Sala-COVID-19 y Educación Secundaria en España

Francisco Javier Aznar Sala is a Professor at the Department of Sociology and Anthropology, Universidad Católica de Valencia, Spain

Contact Address: fjavier.aznar@ucv.es 